\title{
Return to play after anterior cruciate ligament reconstruction: trans-tibial versus antero-medial technique
}

\author{
A. Tucciarone ${ }^{1}$, L. Godente ${ }^{1}$, F. Netti ${ }^{1}$, F. Martinelli ${ }^{3}$, R. Fabbrini ${ }^{1}$, \\ L. Del Ferraro', R. D’Onofrio² \\ 1 Istituto 'Marco Pasquali'-ICOT, Latina, Italy. \\ 2 Scientific Society of Sports Rehabilitation and Posturology, Lazio, Italy. \\ 3 Università degli studi di Roma - Sapienza - Dipartimento di Ortopedia e Traumatologia, Roma, Italy
}

\author{
CORRESPONDING AUTHOR: \\ Agostino Tucciarone \\ Department of Orthopaedic knee and \\ shoulder surgery \\ Istituto "Marco Pasquali" - ICOT \\ Via Franco Faggiana, 1668 \\ Latina - Italy \\ Phone: +390773651490 \\ E-mail: agostinotucciarone@gmail.com
}

DOI:

10.32098/mltj.01.2019.14

\begin{abstract}
SUMMARY
Background. The purpose of the study is to compare clinical outcomes and return to sport between transtibial and anteromedial portal techniques for Anterior Cruciate Ligament (ACL) reconstruction by using single bundle hamstrings grafts secured with variable suspensory fixation device. Methods. Patients undergoing ACL reconstruction were chosen randomly in the period between January 2016 and July 2016. A total of 20 patients met the criteria required to be included in the case study and ultimately grouped by different surgery approaches: 10 transtibial cohort (TT), 10 anteromedial portal cohort (AM). Outcomes were assessed with KT-1000, KSS, KOOS, Tegner-Lysholm scores and Tegner Activity Level scale. Criteria developed by Illingworth et al. were used in the radiological evaluation of bone tunnel placement as a reference to assure quality standards. Results. There was no significant difference in postoperative KT-1000 measurements between the 2 cohorts. A significant improvement in all scores was observed in both cohort. There was a significant difference between the AM group and the TT group in terms of anatomical placement of the femoral tunnels and grafts $(\mathrm{p}<0.001)$. Conclusion. In terms of clinical and functional outcomes, there was no difference between the two groups in the long-term period, except for the time to return sports, which was 6.8 and 7.9 months in the AM and TT groups, respectively.
\end{abstract}

KEY WORDS

arthroscopy; hamstrings; athletes; surgery; knee

\section{INTRODUCTION}

Numerous studies have shown the efficacy of the anatomic anterior cruciate ligament (ACL) reconstruction in restoring normal kinematics and postoperative function of the knee. The purpose of anatomic reconstruction is to place the ACL graft at a more anatomic location on both the tibia and femur. In fact, placing the femoral tunnel in the anatomical femoral footprint of the ACL results in a closer knee joint kinematics, closer to the intact knee than a tunnel suited for the best graft isometry (1). If the graft is placed anterior to this position, it will be tight in flexion, thus restricting the full ROM. If the graft is placed posterior to this position, instead, the graft will tighten with knee extension (2).

Transtibial (TT) technique is a widely used technique for arthroscopic ACL reconstruction that is straightforward and time sparing. In some cases, this technique fails to accurately position femoral and tibial tunnels within the native ACL insertion site (Steiner 2009, Seon 2011), with the consequence that it doesn't restore the normal functions of the knee.

Transtibial tunnel drilling often results in nonanatomic placement of the femoral tunnels (3). In recent years, the transtibial tunnel drilling has been increasingly replaced by anatomic femoral tunnel. As such, it's believed that tunnels offer increased rotational stability, as well as translational and tensioning patterns which are similar to the native ACL (4). Moreover, there is evidence in the literature about the high revision rates in non-anatomical placement of ACL (5), and this could represent inadequacy of transtibial tunnel drilling technique for placement within the native femoral and tibial footprints (6-7). The most common cause of clini- 
cal failure after ACL reconstruction has been cited as incorrect tunnel placement (8-11). It has often been said that TT femoral tunnel drilling does not reach the anatomic site of the ACL insertion but, rather, a high tunnel position $(6,12)$. A lower and more horizontally-oriented placement of the graft on the medial wall of the lateral femoral condyle has been described to obtain a more anatomic femoral tunnel, optimizing rotatory and translational stability. The anteromedial (AM) drilling technique offers such advantages by allowing the drilling of the femoral tunnel independent of the tibial tunnel (13). The biomechanical basis for this achievement is given by Bowers et al. (14), who concluded as follows: when a tibial tunnel is drilled without the need to accommodate subsequent femoral tunnel reaming, more accurate tibial and femoral tunnel positions and resultant sagittal graft obliquity can be achieved.

Anteromedial portal technique has grown in popularity in recent years (15-17) because of the main advantage of drilling the femoral tunnel independently from the tibial tunnel. In this sense, the femoral and tibial tunnels could be placed in the footprints of ACL. However, the quest for anatomic tunnel placement with the AM drilling technique is not without its complications (18). In fact, the potential risks of posterior wall blowout and damage of the medial femoral condyle associating with AM drilling have been well described (18-21). In general, AM portal technique should provide better anatomical bone tunnel placement than transtibial technique, but the superiority of this technique in terms of clinical outcomes is unclear (20).

The purpose of this study is to describe and analyze the evolution of a cohort of patients undergoing ACL reconstruction in terms of radiological, clinical and functional outcomes, and ultimately to compare the results observed between the transtibial and anteromedial portal technique.

\section{MATERIALS AND METHODS}

The present paper is a randomized controlled trial of consecutive patients with isolated ACL deficiency developed at our Institution between January and July 2016, and divided based on the femoral tunnel drilling technique adopted during the reconstruction. The study meets the ethical standard of the journal (22). It was performed after obtaining the approval of the local ethics committee and informed signed consent of every participant.

Inclusion criteria were as follows: isolated primary ACL injury; male sex; healthy contralateral knee. Exclusion criteria were as follows: previous surgery on the affected limb; open physes on the femur and/or tibia; associated capsular and meniscal lesions of any type; associated chondral lesion of grade III to IV according to ICRS classification; smoking habit; obesity (BMI higher than 30); intra- and peri-articular lesions on the contralateral limb; early osteoarthritis; endocrine, collagen, neurological, infective, and rheumatological pathologies.

Once the patient was eligible for the study, he/she was anonymized with a random number, and $\mathrm{Ms}$ Excel $^{\circledR}$ Random Formula was used successively to assign patients to Study (AM technique) or Control (TT technique) Group.

All patients were operated by a single senior surgeon (TA), reconstructing the ruptured ACL with quadruple-stranded hamstring auto-grafts (gracilis and semitendinosus). A standard technique was performed by alternating a TT or an AM femoral tunnel drilling technique according to the group in which the patient was included. The same rehabilitation protocol was used in both groups.

Patient evaluation was performed at the time of inclusion in the study, at the first, third, sixth, ninth months, and at the final 1 year follow-up, for clinical, subjective, and radiographic outcomes. The Lachman test was used to evaluate the anterior-posterior stability of the knee, and the Pivot shift test to evaluate the rotational stability of the knee, along with KT-1000 (MEDmetric, San Diego, CA) evaluation for a more quantitative definition of the residual translation. Four hop tests (single, crossover, and triple hops for distance, and a $6 \mathrm{~m}$ timed hop) were used for the functional assessment. VAS, IKDC, KSS, KOOS and Tegner-Lysholm scoring scales were used for subjective satisfaction definition. Tegner Activity Level Scale was used to evaluate the return to sport, with the motion analysis through camera shooting.

Radiological outcomes were assessed using the criteria developed by lllingworth et al. (23). These criteria were used to define whether ACL reconstruction fell outside an anatomical range or not. Accordingly, the femoral tunnel angle was measured on the anterior-posterior radiography of the knee at $45^{\circ}$ flexion, and the inclination angle of the graft was measured in sagittal sections of magnetic resonance imaging (MRI) of the knee at extension. When the femoral tunnel became less than $33.9^{\circ}$ and the inclination angle became greater than $54.5^{\circ}$, it was considered that ACL reconstruction fell outside an anatomical range.

Once data were gathered from the final follow-up, tests were performed to analyzed differences in the two groups, and correlations with the other results observed.

\section{RESULTS}

A total of 20 patients constituted the sample size. They were randomly assigned to anteromedial (AM) technique group (10 patients) or transtibial (TT) technique group (10 patients) after the screening with inclusion and exclusion criteria. All patients were semi-professional athletes. 
No differences in demographic characteristics were observed between the cohorts, limiting inclusion bias to a minimum. Data were collected over a 1 year period; no statistically significant differences were observed at the intermediate follow-up, therefore only the final outcomes are reported. In the AM cohort, KT-1000 measurements ranged from 0 to $3 \mathrm{~mm}$, whereas in the TT group, the measurements ranged from 0 to $5 \mathrm{~mm}$. There was no significant difference between the 2 cohorts (STAT) in postoperative Lachman tests, pivot shift-signs tests and KT-1000 measurements.

With available data, significant differences were not to be found between the cohorts for any postoperative score measured $(\mathrm{p}>2)$ in each follow up examination. Also, VAS, IKDC, KSS, KOOS and Tegner-Lysholm scales were similar between both groups (Student t, p > 0.05).

No patient required revision of ACL reconstruction.

There was an episode of wound dehiscence in AM group treated with surgical wound revision.

ACL reconstruction fell within an anatomical range in 9 patients $(90 \%)$ in the AM group (figure 1) and in 4 patients $(40 \%)$ in the TT group (figure 2 ). Statistically significant difference was observed between the two groups (Chi squared, p < 0.001) (Table I, II).

The time to return to play was 6.8 months (range 69) and 7.9 months (range 7-10) in the AM and TT groups, respectively (Student $t, p>0.05$ ). The results obtained analyzing the TegnerActvity Level Scale at one year after surgery, show that $10 \%$ of the patients reached level 7, 70\% level 8 and $20 \%$ level 9 of activity in TT group, while in AM group, $10 \%$ of patients reached level 8 and $90 \%$ level 9 . Statistically significant difference was observed between the two groups (Chi squared, p < 0.05) (table I, II).

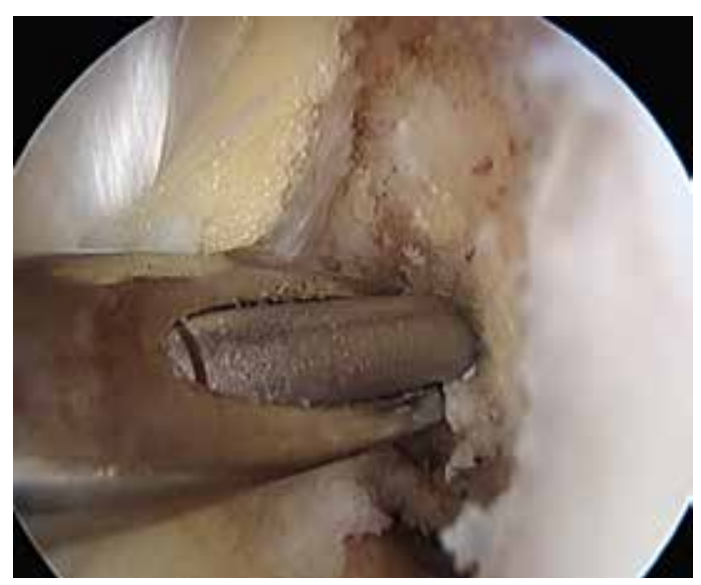

Figure 1

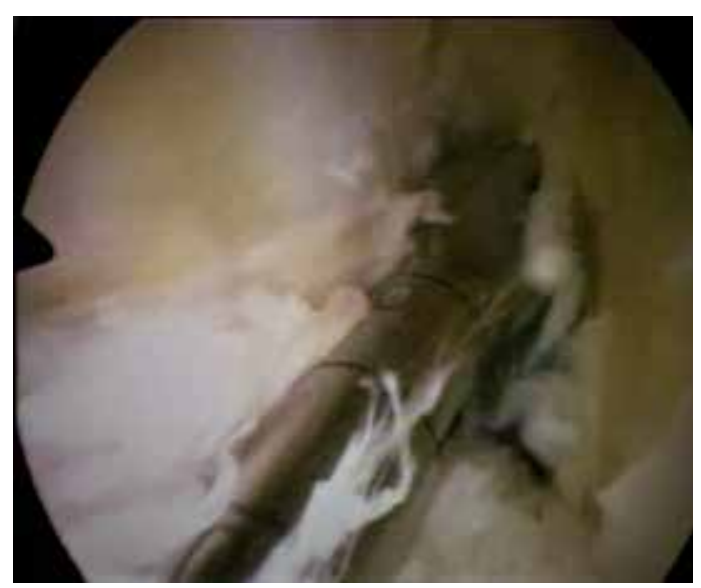

Figure 2

Table I - Transtibial technique, one year follow up.

\begin{tabular}{lccccc}
\hline & $\begin{array}{c}\text { KT-1000 } \\
(\mathbf{m m})\end{array}$ & $\begin{array}{c}\text { femoral tunnel angle } \\
\left(\mathbf{4 5 ^ { \circ }} \mathbf{R X}\right)\end{array}$ & $\begin{array}{c}\text { inclination angle of } \\
\text { the graft } \\
(\mathbf{R M})\end{array}$ & $\begin{array}{c}\text { Tegner Activity Level Scale } \\
\text { (one year) }\end{array}$ & $\begin{array}{c}\text { return to play } \\
(\mathbf{m o n t h})\end{array}$ \\
\hline P1 & 3 & $\mathbf{2 8 . 9}$ & $\mathbf{6 4 . 1}$ & 8 & 7 \\
\hline P2 & 1 & 39.2 & 50.8 & 8 & 7 \\
\hline P3 & 4 & $\mathbf{1 7 . 6}$ & $\mathbf{6 2 . 3}$ & 7 & 10 \\
\hline P4 & 0 & $\mathbf{2 1 . 8}$ & $\mathbf{6 8 . 4}$ & 8 & 9 \\
\hline P5 & 2 & 35.2 & 47.6 & 9 & 7 \\
\hline P6 & 3 & 31.1 & $\mathbf{5 8 . 9}$ & 8 & 7 \\
\hline P7 & 3 & 37.6 & 52.3 & 8 & 8 \\
\hline P8 & 5 & $\mathbf{2 9 . 2}$ & $\mathbf{5 7 . 3}$ & 8 & 7 \\
\hline P9 & 2 & 34.1 & 49.9 & 8 & 9 \\
\hline P10 & 4 & $\mathbf{2 3 . 4}$ & $\mathbf{5 9 . 5}$ & & $\mathbf{7 . 9}$ \\
\hline Average & $\mathbf{2 . 7}$ & $\mathbf{2 9 . 8 1}$ & $\mathbf{5 7 . 1 1}$ & & 7 \\
\hline
\end{tabular}


Table II - Anteromedial technique, one year follow up.

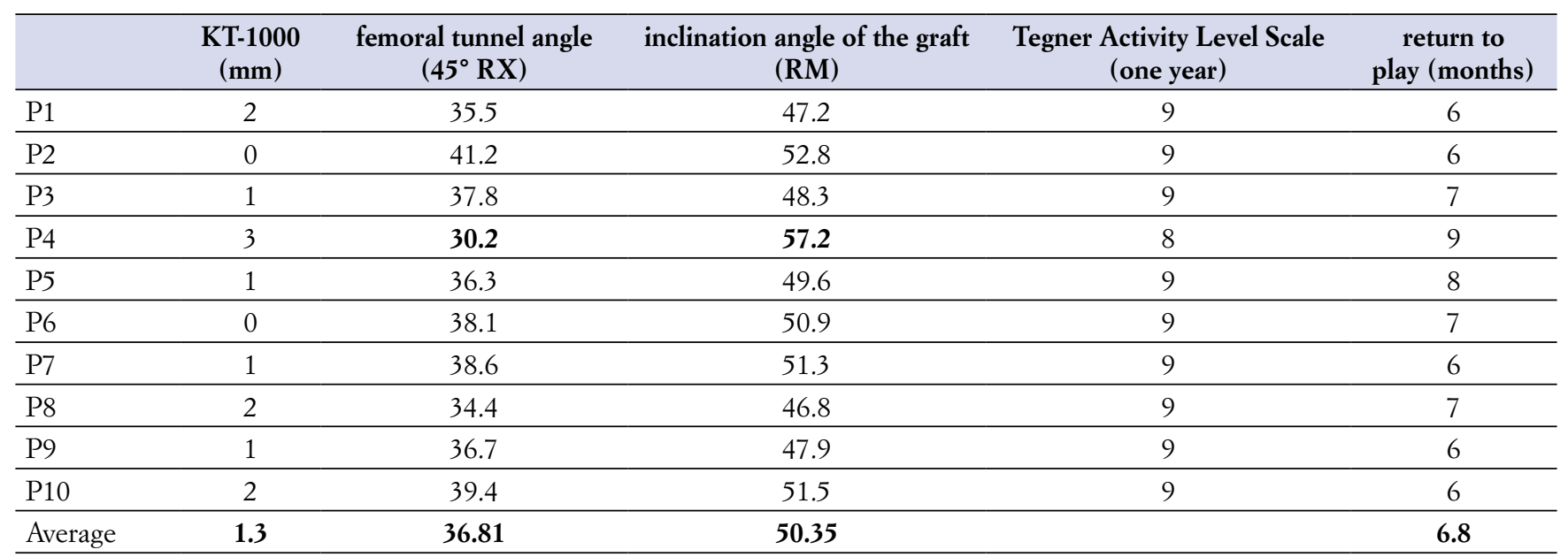

\section{DISCUSSION}

This study compared the AM and TT techniques in ACL reconstruction. It was observed that the AM technique was superior to the TT technique in providing ACL reconstruction within anatomical range $(\mathrm{p}<0.001)$ (figure 3,4$)$. The patients in the AM group returned to sports activity 1.1 months earlier when compared to TT group ( $<<0.001$ ).

Although the difference between both groups was not statistically significant in terms of pivot shift test, the percentage of abnormal pivot shift was higher in the TT group when compared to AM group ( $20 \%$ and $0 \%$, respectively). The present finding are in line with the most recent literature.

Numerous studies have shown the biomechanical advantages of anatomic femoral tunnel positioning. However, it's still being discussed what is the best technique to use for accurately placing the ACL graft in an anatomic location on both tibia and femur.

Using a modification of the conventional TT approach and drilling the femoral tunnel independently through the standard AM portal are two commonly described techniques.

Modifications of the TT technique have been proposed in an attempt to improve the angle of the femoral tunnel, and to better restore the femoral ACL footprint. Despite these modifications, some concerns still exist about the ability to restore the native femoral ACL insertion through a TT approach. Dargel et al. have found that the use of the TT technique tends to position the femoral tunnel toward the roof of the notch and anterior to the ACL footprint. Paessler et al. showed that femoral tunnel drilling using the TT technique did not create anatomic positioning of the ACL, which could be the reason why the stability of the knee is not adequately restored.
Bedi et al. have shown the biomechanical superiority of ACL reconstruction by using the AM portal technique versus the conventional TT approach. The AM portal reconstruction resulted in Lachman examination findings equivalent to the uninjured knee whereas the Lachman examination findings after TT reconstruction could not be differentiated from the ACL-deficient condition. The AM portal reconstruction produced significantly more translational restraint during manual and instrumented pivot-shift examination, when compared with both the native ACL and TT ACL reconstruction.

In his study, Chen et al. have found that the AM technique yielded superior outcomes in terms of Lachman test, pivotshift test, and IKDC scores. In addition, AM technique yielded comparable outcomes in terms of Lysholm scores as well. Similarly to our results, the AM technique did not yield any inferior outcomes across all the comparative parameters studied. Same results were observed by Koutras et al, concluding that the AM technique is superior to the TT technique in providing anatomical ACL reconstruction and return to sport activities. However, there was no difference between the groups in the early and long period in terms of the Lachman test, pivot shift-sign test, KSS, KOOS, and Tegner-Lysholm scores.

AM technique provides significant improvement in postoperative ACL stability that is clinically important. In terms of KT-1000 testing, 3 of 4 articles analyzed in a systematic review showed superior results in the AM group, although the absolute differences in measurements were small. Moreover, in a recent meta-analysis, the $\mathrm{AM}$ technique was found to improve clinical outcomes of ACL reconstruction compared with the TT technique: ACL reconstruction 
using the AM technique is associated with improved postoperative knee stability, while the functional outcomes of the AM technique are at least comparable with those of the TT technique (24).

Lastly, in the assessment of tunnel and graft placement, Illingworth et al. (23) confirmed with 3D computed tomography that a femoral tunnel angle less than $33.9^{\circ}(100 \%$ sensitivity and $85 \%$ specificity) on the anteroposterior $x$-ray of the knee and an inclination angle more than $54.5^{\circ}(100 \%$ sensitivity and $87.5 \%$ specificity) on the sagittal MRI is an effective method to determine whether the ACL reconstruction is within the anatomical ranges or not. Our results showed a significantly better anatomical tunnel positioning for the AM technique. This finding could be easily explained by the possibility to drill a completely independent femoral tunnel, which is perfectly centered in the femoral ACL footprint. Associated with the comparability of the other parameters, this advantage should let the AM technique be preferred for ACL reconstruction.

To date medical literature emphasizes as follows:

a) about $76-95 \%$ of athletes undergoing LCA reconstruction return to pre injury performance levels after 12 months (25);

b) re-injury rate is increased by $23 \%$.

Supported by strong Scientific Evidence, we can underline how a not solved LCA injury caused by "functional-postural damage" can be a factor of poor "compliance", and consequently a decrease in athletic performance levels during the Reconditioning phase.

During Step of Movement Analysis, we could additionally highlight an adaptive dynamic valgus during athletic performance. Associated with a decrease in rotational expression control, this dynamic valgus correlates with an increased risk of LCA reinjury. Observed during a specific sport-related movement, the increase of the knee valgus often suggests the inability of the quadriceps/flexor muscle group (specifically, sartorius, gracilis, and semitendinosus) to control and oppose the valgus movement. The phenomenon is clearly perceptible during the landing phase of a jump, or when the athlete is performing the cutting maneuver.

We can point out that the co-contraction of crural, quadriceps and gastrocnemious is the fulcrum of muscle activity, especially in complex football gesture expression. The simultaneous contraction of quadriceps and crural muscles plays a role of maximum protection and stability of the knee during the various technical / tactical situations.

In both football or basketball matches, unidirectional straight run is a minor percentage compared to lateral run. Performed at different speeds, torsional movements require a greater neuromuscular control. Combined with internal /

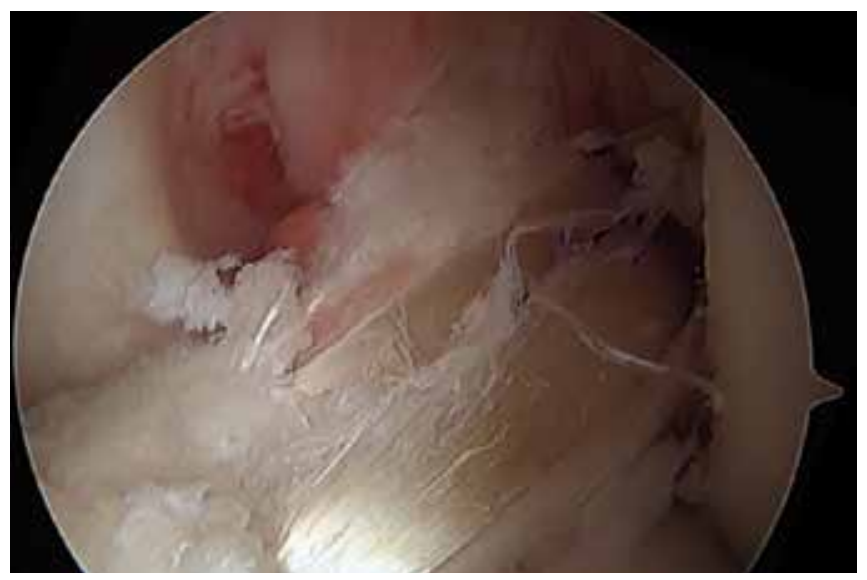

Figure 3

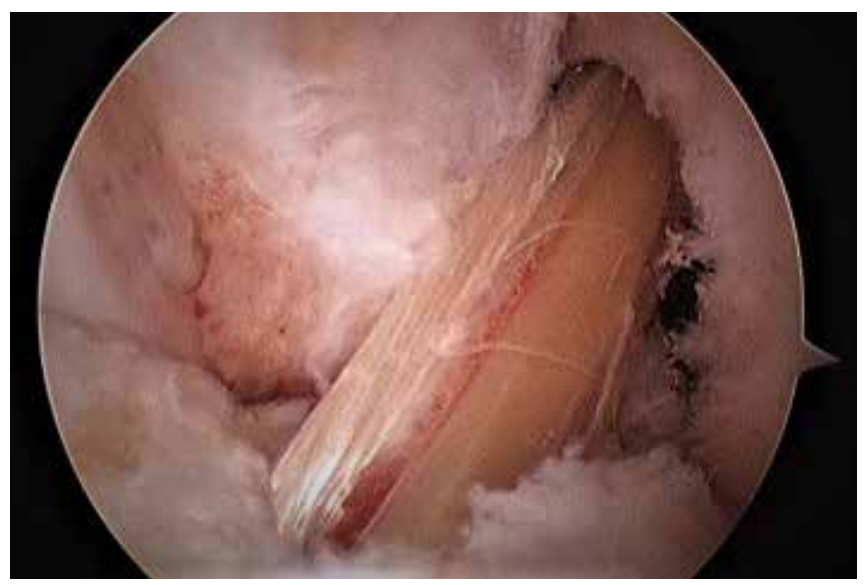

Figure 4

external rotation on the knee, varo-valgus movements create high-risk "movements" of non-contact injuries of the LCA. In the planning of the reconditioning phase, all these statements should be considered.

Differences of strength are observed above all the kinetic chains. Wrong static and dynamic compensation could lead to complication in returning to sports.

Repetitive gestural movements lead to postural long-term changes in kinematics, which can result in "altered motion patterns" that ultimately will determine pathological functional sequences. An altered "muscular tension" sequence can increase the risk of reinjury with poor performance levels shortening the career of the athletes.

The assessment of asymmetrical sports-related gestures, the choice of therapeutic exercises, as well as the neuromuscular training programs designed to modulate the control of the adaptive knee valgus, all these actions should thus be 
taken as an effective measure to study each athlete as an individual case.

Particularly, we are currently re-analyzing the posture / functional aspects during the rehabilitation and reconditioning phase, to optimize the return to the performance levels prior to reconstructive surgery and reduce the risk of "compliance" and reinjury.

\section{CONCLUSION}

In conclusion, the importance of an anatomical tunnel placement is established, and the AM technique was superior to the TT technique in providing the anatomical placement of the graft and regarding time to return sports in ACL reconstruction. However, there was no significant difference

\section{REFERENCES}

1. Plakseychuk A, VanScyoc A, Sasaki T, Debski RE, McMahon PJ, Fu FH. Varying femoral tunnels between the anatomical footprint and isometric positions: effect on kinematics of the anterior cruciate ligament-reconstructed knee. Am J Sports Med 2005; 33:712-718.

2. Hefzy MS, Grood ES, Noyes FR. Factors affecting the region of most isometric femoral attachments. Part II: The anterior cruciate ligament. Am J Sports Med 1989; 17:208-216.

3. Strauss EJ, Barker JU, McGill K, Cole BJ, Bach BR, Verma NN. Can anatomic femoral tunnel placement be achieved using a transtibial technique for hamstring anterior cruciate ligament reconstruction? Am J Sports Med 2011; 39:12631269.

4. Lee MC, Seong SC, Lee S, Chang CB, Park YK, Jo H, Kim $\mathrm{CH}$. Vertical femoral tunnel placement results in rotational knee laxity after anterior cruciate ligament reconstruction. Arthroscopy 2007; 23:771-778.

5. Marchant BG, Noyes FR, Barber-Westin SD, Fleckenstein C. Prevalence of nonanatomical graft placement in a series of failed anterior cruciate ligament reconstructions. Am J Sports Med 2010; 38:1987-1996.

6. Abebe ES, Moorman CT, Dziedzic TS, Spritzer CE, Cothran RL, Taylor DC, Garrett WE, DeFrate LE. Femoral tunnel placement during anterior cruciate ligament reconstruction: an in vivo imaging analysis comparing transtibial and 2 -incision tibial tunnel independent techniques. Am J Sports Med 2009; 37:1904-1911.

7. Alentorn-Geli E, Samitier G, Alvarez P, Steinbacher G, Cugat R. Anteromedial portal versus transtibial drilling techniques in ACL reconstruction: a blinded cross-sectional study at two. to five-year follow-up. IntOrthop 2010; 34:747-754.

8. Marchant BG, Noyes FR, Barber-Westin SD, Fleckenstein C. Prevalence of nonanatomical graft placement in a series of failed anterior cruciate ligament reconstructions. Am J Sports Med 2010; 38:1987-1996.

9. Diamantopoulos AP, Lorbach O, Paessler HH. Anterior cruciate ligament revision reconstruction: Results in 107 patients. Am J Sports Med 2008; 36:851-860. between the groups in the early period in terms of the clinical outcomes. Definite clinical and functional data, useful to determine objectively the superiority of a technique over the other, are currently still lacking.

Associated with the recent literature, the result of this paper seem to advise for an AM tunnel drilling in order to obtain the good-to-excellent clinical outcomes. The latter are usually associated with the TT technique but allow for a more anatomical reconstruction, which is biomechanically superior. A proper randomized clinical trial is still needed, although, to clearly demonstrate which technique is definitely superior.

\section{Disclosure}

No conflicts of interest were declared by the authors.

10. Loh JC, Fukuda Y, Tsuda E, Steadman RJ, Fu FH, Woo SL. Knee stability and graft function following anterior cruciate ligament reconstruction: Comparison between 11 o'clock and 10 o'clock femoral tunnel placement. Arthroscopy 2003; 19:297-304.

11. Noh JH, Roh YH, Yang BG, Yi SR, Lee SY. Femoral tunnel position on conventional magnetic resonance imaging after anterior cruciate ligament reconstruction in young men: Transtibial technique versus anteromedial portal technique. Arthroscopy 2013; 29:882-890.

12. Arnold MP, Kooloos J, van Kampen A. Single-incision technique misses the anatomical femoral anterior cruciate ligament insertion: A cadaver study. Knee Surg Sports Traumatol Arthrosc 2001; 9:194-199.

13. Steiner ME. Independent drilling of tibial and femoral tunnels in anterior cruciate ligament reconstruction. J Knee Surg 2009; 22:171-176.

14. Bowers AL, Bedi A, Lipman JD, et al. Comparison of anterior cruciate ligament tunnel position and graft obliquity with transtibial and anteromedial portal femoral tunnel reaming techniques using high resolution magnetic resonance imaging. Arthroscopy 2011; 27:1511-1522.

15. Chalmers PN, Mall NA, Cole BJ, Verma NN, Bush-Joseph CA, Bach BR Jr. Anteromedial versus transtibial tunnel drilling in anterior cruciate ligament reconstructions: A systematic review. Arthroscopy 2013; 29:1235-1242.

16. Hussein M, van Eck CF, Cretnik A, Dinevski D, Fu FH. Prospective randomized clinical evaluation of conventional single-bundle, anatomic single-bundle, and anatomic double-bundle anterior cruciate ligament reconstruction: 281 cases with 3. to 5-year follow-up. Am J Sports Med 2012; 40:512-520.

17. Riboh JC, Hasselblad V, Godin JA, Mather RC III. Transtibial versus independent drilling techniques for anterior cruciate ligament reconstruction: A systematic review, meta-analysis, and meta-regression. Am J Sports Med 2013; 41:2693-2702.

18. Lubowitz JH. Anteromedial portal technique for the anterior cruciate ligament femoral socket: Pitfalls and solutions. Arthroscopy 2009; 25:95-101. 
19. Bedi A, Raphael B, Maderazo A, Pavlov H, Williams RJ III. Transtibial versus anteromedial portal drilling for anterior cruciate ligament reconstruction: A cadaveric study of femoral tunnel length and obliquity. Arthroscopy 2010; 26:342-350.

20. Tudisco C, Bisicchia S, Cosentino A, Chiozzi F, Piva M. Knee stability, athletic performance and sport-specific tasks in non-professional soccer player after ACL reconstruction: comparing Trans-tibial and Anteromedial portal techniques. Muscles, Ligaments and Tendons J 2015; 5(3):175-180.

21. Dave LY, Nyland J, Caborn DN. Knee flexion angle is more important than guidewire type in preventing posterior femoral cortex blowout: A cadaveric study. Arthroscopy 2012; 28:1381-1387.

22. Padulo J, Oliva F, Frizziero A, Maffulli N, Muscles, Ligaments and Tendons Journal. Basic principles and recommendations in clinical and field science research: 2016 update. MLTJ 2016; 6(1):1-5.

23. Illingworth KD, Hensler D, Working ZM, Macalena JA, Tashman $\mathrm{S}$ and $\mathrm{Fu} \mathrm{FH}$. A Simple Evaluation of Anterior Cruciate Ligament Femoral Tunnel Position: The Inclination Angle and Femoral Tunnel Angle. Am J Sports Med 2011;39:2611. Originally published online September 9, 2011.

24. Chen Y, Chua KHZ, Singh A, Tan JH, Chen X, Lingaraj K. Outcome of Single-Bundle Hamstring Anterior Cruciate Ligament Reconstruction Using the Anteromedial Versus Transitbial Technique: A Systematic Review and Meta-analysis. Arthroscopy 2015; 31(9):1784-1794.

25. Notarnicola A, Maccagnano G, Barletta F, et al. Returning to sport after anterior cruciate ligament reconstruction in amateur sports men: a retrospective study. Muscles, Ligaments and Tendons J 2016; 6(4): 486-491. 